\title{
Efficient Target Detection from Hyperspectral Images Based On Removal of Signal Independent and Signal Dependent Noise
}

\author{
Poornima.P ${ }^{1}$, Chitra valavan ${ }^{2}$ \\ ${ }^{1-2}$ Department Of Electronics And Communication Engineering,AVC College Of Engineering, India
}

\begin{abstract}
In our proposed system the random noise present in hyper spectral image is removed by means of tensor based decomposition methods. The noises present in hyper spectral images are classified into two categories namely: signal independent noise and signal dependent noise. The noises present in the hyper spectral images have dependence on the noise variance of the signal. The input image is separated into seven different frequency bands and noise is added into some of those bands. The corresponding peak signal to noise ratio was calculated for each band based on mean square error value. In our project we are using GUI tool in $M A T L A B$ to enable user friendly approach in noise removal. The overall system comprises of three types of algorithm namely: parallel factor analysis (PARAFAC), hyper spectral noise estimation (HYNE) and multidimensional Wiener filter (MWF).The first one, named as the PARAFACSI-PARAFACSD method, uses a multi linear algebra model, PARAFAC decomposition, twice to remove SI and SD noise, respectively. The second one is a combination of the multiple-linear-regression-based approach termed as the HYNE method and PARAFAC decomposition, which is named as the HYNE-PARAFAC method. The last one combines the MWF method and PARAFAC decomposition and is named as the MWF-PARAFAC method. SI noise is removed from the original image by PARAFAC decomposition, HYNE method, or MWF method based on the property of SI Noise. SD components can be further reduced by PARAFAC decomposition due to its own statistical property.
\end{abstract}

Keywords: Parafac, Mwf, Hyne, Si Noise, Hyperspectral Image.

\section{Introduction}

For much of the past decade, hyper-spectral imaging has been an area of active research and development, and hyper-spectral images have been available only to researchers. With the recent appearance of commercial airborne hyper-spectral imaging systems, hyper-spectral imaging is poised to enter the mainstream of remote sensing. Hyper-spectral images will find many applications in resource management, agriculture, mineral exploration, and environmental monitoring. But effective use of hyper-spectral images requires an understanding of the nature and limitations of the data and of various strategies for processing and interpreting it. The "hyper" in hyper spectral means "over" as in "too many" and refers to the large number of measured wavelength bands. Hyper spectral images are spectrally over determined, which means that they provide ample spectral information to identify and distinguish spectrally unique materials. Hyper spectral imagery provides the potential for more accurate and detailed information extraction than possible with any other type of remotely sensed data.The noise in HSIs can be distinguished into two classes random noise and fixed-pattern noise. Photon and thermal noise are examples of random noise in HSIs, whereas striping, periodic, and interference noise are examples of fixed-pattern noise, which is generated by errors in the calibration process and can be removed from the HSI by suitable procedures. However, random noise, due to its stochastic nature, cannot be removed by those procedures and influences the performance of the algorithms adopted in hyper-spectral data exploitation. For sensors used in hyper-spectral imagery, the theory predicts that random noise mainly comes from two aspects: signal-independent (SI) electronic noise and signal-dependent (SD) photonic noise[1].

\section{A. Proposed system:}

\section{System Architecture}

In our proposed system we design new approach removing noise in HSI image. Using hybrid of algorithms namely Hyper-spectral noise estimation, Parallel factor analysis and Multi-dimensional Wiener filter. Its design is based on eliminating the existing methods drawbacks and removal of the SI white noise, SI color noise and SD noise from the HSI with high degree of accuracy. The SI white noise is the Gaussian white Noise and SI color noise is random variable noise. The white Gaussian noise adds white pixel in the image leads to low density and low lighting. The random noise randomly adds black and white pixels caused by electrical circuit and SD noise caused by photonic noise of the circuit. The SI white noise will be removed by using hybrid of PARAFAC, HYNE, and MWF. Then the random noise will be removed by using the combination of PARAFAC and HYNE methods. Then the SD noise will be removed by using PARAFAC decomposition method. All types of noise are having major impact on the hyper-spectral image due to that reasons the researchers concentrate on the removal of those noises and getting accurate hyper-spectral image as possible. 
All of the previous noise removal processes are done by means of filters but the filters removed only any one of the noise. But it is desired that all the noises will need to be removed to get the best result by getting high quality image. In that cause we design a new hybrid method for de-noising purpose. In that de-noising process we remove white noise by using MWF. The image noise is estimated by using HYNE method it is a type of multi linear algebra algorithm. Then we are using PARAFAC analysis to decompose the image and MWF is used to remove the SI white noise. After that the SI color noise is removed by decomposing the SI noise removed image by estimating that noise by Hyper-spectral noise estimation and decompose it with the Parallel Factor analysis to remove the SI color noise and the output image will contains only the SD noise then that noise will be removed by using PARAFAC decomposition.

\section{B. Flow diagram}

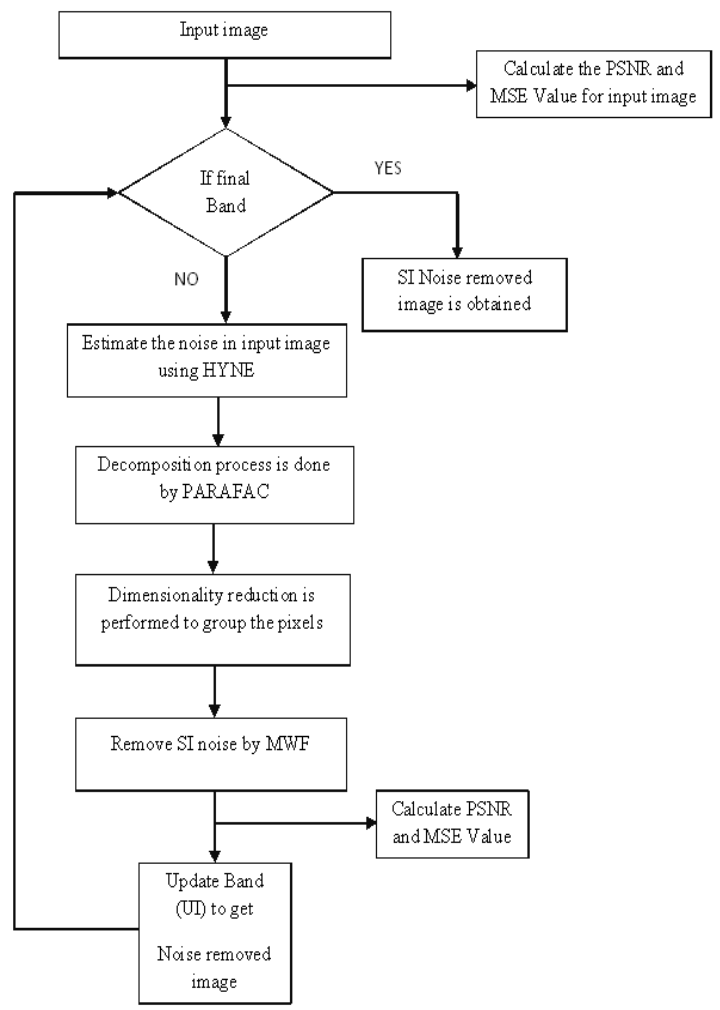

Fig. 1 flow diagram

\section{A. Modules description:}

III. Overall Architecture

\section{1) Addition of noise:}

The original input image is noiseless image because in real world all the digital image is noise free image. So that reason we add the noise in input image. In our proposed we added three types of noise they are SI white noise, SI color noise, SD noise. The SI color noise [2]in real world that created by the electronic circuits. The SD noise in real world cause of Photonic noise. The property of those noise is add randomly added the image pixels. In real world the SI white Gaussian noise crated by low lighting and low brightness. The property of the white Gaussian noise is adding white pixels in image pixels. In proposed method we add noise in image based on that properties. After adding the noise we calculate the PSNR value to estimate the noise level in the input image.

\section{2) Steps involved in Noise removal:}

The three types of noise will be removed based on the hybrid of three different types of method of HYNE, PARAFAC, and MWF. That method combination of different manner it remove different type of images. 
Hyne:

The HYNE means Hyper spectral noise estimation method. The HYNE method is based on the Multi linear Regression (MLR).

MLR is used as common basis for three analysis methods used in The Unscramble:

- Multiple Linear Regressions;

- Analysis of Effects;

- Response Surface Analysis

The Decomposition algorithm is the most widely used algorithm to compute the estimated regression coefficients for MLRHyperspectral sensors measure the radiance from the observed scene in many spectral bands very close in wavelengths; thus, the signal $\mathrm{X}$ is generally characterized as having strong spectral correlation. MLR-based approach that exploits the strong spectral correlation of the signal and the weak between band correlations of the SI noise in HSI. After estimating the pixel values in each band as a linear combination of the pixel values in the remaining bands, the noise is reduced as a result.

\section{Parafac:}

Parallel factor analysis extends the ideas and methods of standard two-way factor analysis to three way data. The tri-linear model underlying PARAFAC[2] may be the simplest nontrivial way to extend the algebra of the factor analysis model from two-way to three way data.

$$
x_{i j}=a_{i 1} f_{j 1}+a_{i 2} f_{j 2}+\cdots+a_{i r} f_{j r}+\cdots+a_{i R} f_{j R}+e_{i j}
$$

Where $X_{i, j}$ is the entry in row $i$ and column $\mathrm{j}$ of the $\mathrm{I}$ by $\mathrm{J}$ data matrix $\mathrm{X}$.

Factor analysis is a statistical method used to describe variability among observed, correlated variables in terms of a potentially lower number of unobserved variables called factors. For example, it is possible that variations in four observed variables mainly reflect the variations in two unobserved variables. Factor analysis searches for such joint variations in response to unobserved latent variables. The observed variables are modeled as linear combinations of the potential factors, plus "error" terms. The information gained about the interdependencies between observed variables can be used later to reduce the set of variables in a dataset. Computationally this technique is equivalent to low rank approximation of the matrix of observed variables. Factor analysis originated in psychometrics, and is used in behavioral sciences, social sciences, marketing, product management, operations research, and other applied sciences that deal with large quantities of data. Factor analysis is related to principal component analysis (PCA)[3], but the two are not identical. Latent variable models, including factor analysis, use regression modeling techniques to test hypotheses producing error terms, while PCA is a descriptive statistical technique. There has been significant controversy in the field over the equivalence or otherwise of the two technique.

\section{Multi-dimensional median filter:}

The multi-dimensional median filter is very well filter to remove the white Gaussian noise. Wiener theory, formulated by Norbert Wiener, forms the foundation of data-dependent linear least square error filters. Multi-dimensional Wiener filters play a central role in a wide range of applications such as linear prediction, echo cancellation, signal restoration, channel equalization and system identification. The coefficients of a Multidimensional Wiener filter are calculated to minimize the average squared distance between the filter output and a desired signal. In its basic form, the Wiener theory assumes that the signals are stationary processes. However, if the filter coefficients are periodically recalculated for every block of $\mathrm{N}$ signal samples then the filter adapts itself to the average characteristics of the signals within the blocks and becomes block-adaptive. A blockadaptive (or segment adaptive) filter can be used for signals such as speech and image that may be considered almost stationary over a relatively small block of samples. In this chapter, we study Multi-dimensional Wiener filter theory, and consider alternative methods of formulation of the Multi-dimensional Wiener filter problem. We consider the application of Multi-dimensional Wiener filters in channel equalization, time-delay estimation and additive noise reduction. A case study of the frequency response of a Multi-dimensional Wiener filter, for additive noise reduction, provides useful insight into the operation of the filter. We also deal with some implementation issues of Multi-dimensional Wiener filters. That Filter will remove the white Gaussian noise in the signal data.

\section{3) Removal of SI white Noise:}

In the Hyper-spectral noise removal process starts from the reduction of the SI white noise in the image. The SI white noise reduction technique involves the decomposition of image by using PARAFAC and 
estimate the noise in each pixel by using HYNE method then remove the noise using MWF. After that the SI white noise will be removed then the image contains only the SI color noise and SD noise.

\section{Results}

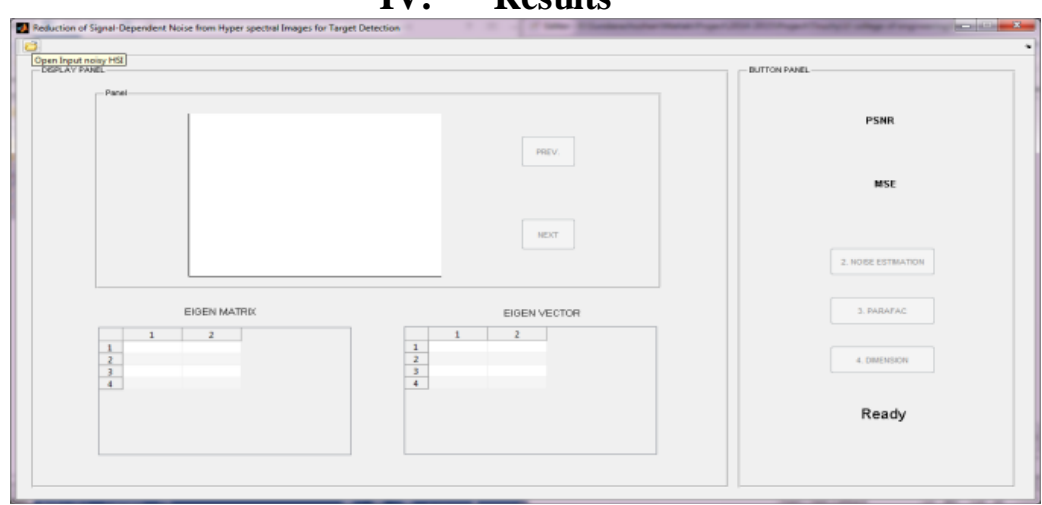

Fig. 2 workspace for noise removal

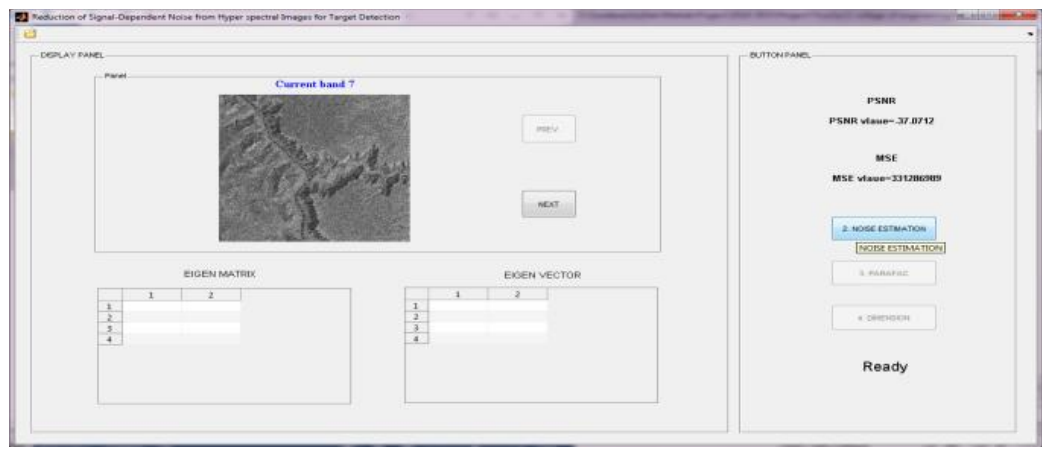

Fig. 3 input image

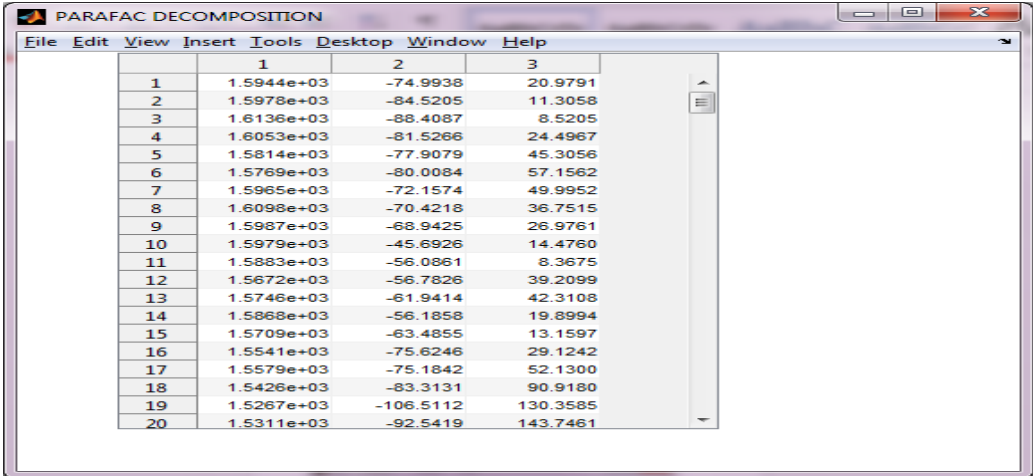

Fig.4 PARAFAC decomposition

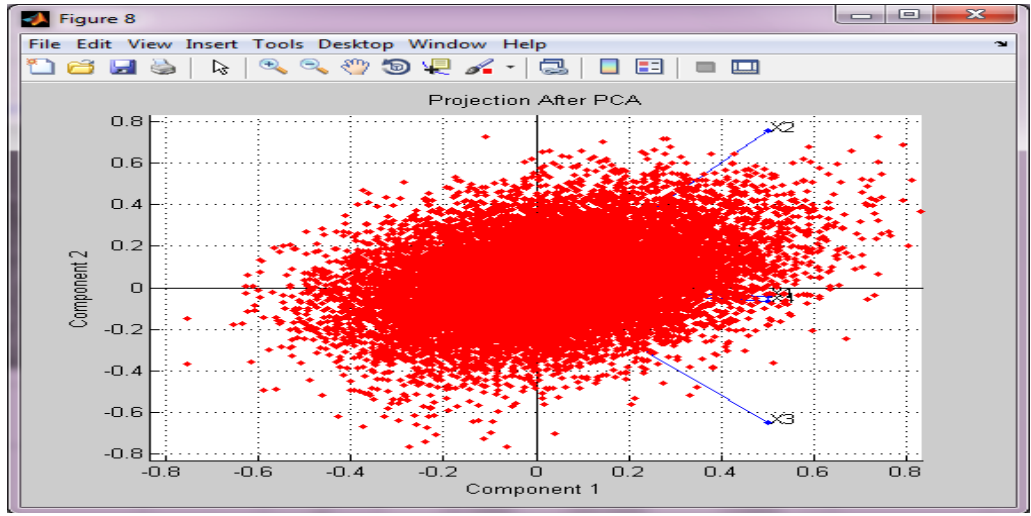

Fig.5 PCA projection 


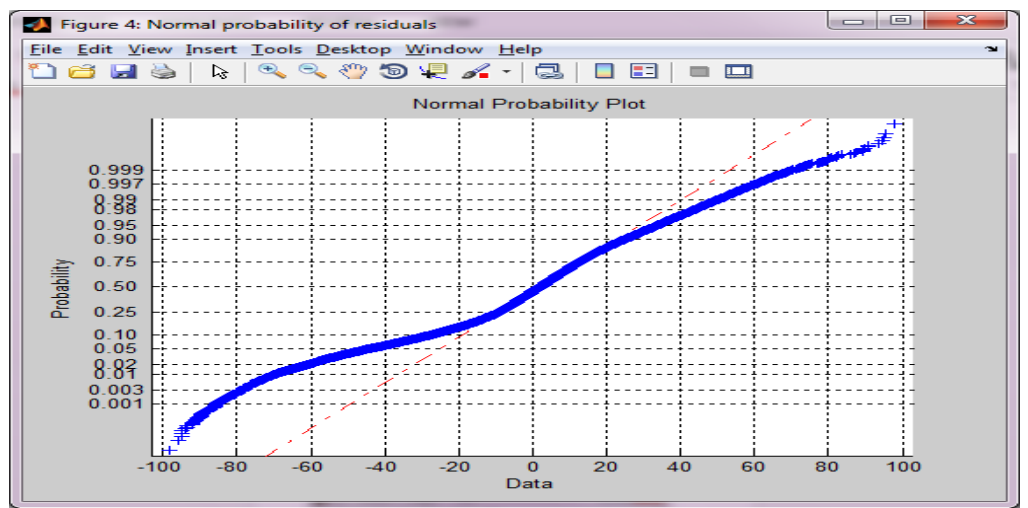

Fig.6 normal probability plot

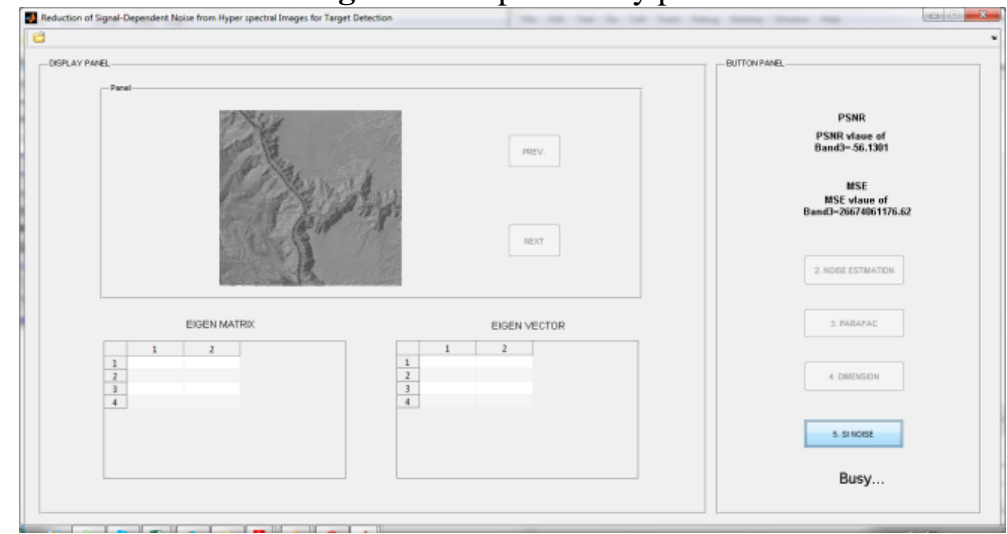

Fig.7 final output

\section{Conclusions}

First, PARAFAC decomposition and HYNE and MWF methods can be applied to remove the SI white noise, or PARAFAC decomposition and the HYNE method can be used to delete the coloured SI noise. Then, to reduce the residual SD components, PARAFAC decomposition is applied to the denoised HSI by the previous step. The performance of the proposed PARAFACSI -PARAFACSD, HYNE-PARAFAC, and MWFPARAFAC methods is validated on the simulated HSIs distorted by both SD and white SI noise and on the realworld HYDICE HSIs.

\section{References}

[1]. C.-I. Chang, Hyperspectral Imaging: Techniques for spectral detection and classification. New York, NY, USA: Kluwer, 2003.

[2]. J. M. P. Nascimento and J. M. Bioucas-Dias, "Hyperspectralunmixing based on mixtures of Dirichlet components," IEEE Trans. Geosci. Remote Sens., vol. 50, no. 3, pp. 863-878, Mar. 2012

[3]. N. Renard and S. Bourennane, "Improvement of target detection methodsbymultiway filtering," IEEE Trans. Geosci. Remote Sens., vol. 46, no. 8, pp. 2407-2417, Aug. 2008.

[4]. R. Archibald and G. Fann, "Feature selection and classification of hyperspectral images with support vector machines," IEEE T rans. Geosci. Remote Sens., vol. 4, no. 4, pp. 674-677, Oct. 2007.

[5]. N. Acito, M. Diani, and G. Corsini, "Signal-dependent noise modeling and model parameter estimation in hyperspectral images," IEEE Trans. Geosci. Remote Sens., vol. 49, no. 8, pp. 2957-2971, Aug. 2011.

[6]. P. Rakwatin, W. Takeuchi, and Y. Yasuoka, "Stripe noise reduction in modis data by combining histogram matching with facet filter,” IEEE Trans. Geosci. Remote Sens., vol. 45, no. 6, pp. 1844-1856, Jun. 2007.

[7]. M. Uss, B. Vozel, V. Lukin, and K. Chehdi, "Local signal-dependent noise variance estimation from hyperspectral textural images," IEEE J. Sel. Topics. Signal Process., vol. 5, no. 3, pp. 469-486, Jun. 2011 